\title{
Insetos na escola: abordagens didáticas sobre os insetos na educação básica
}

\section{Insects at school: teaching approaches to insects in basic education}

Insectos en la escuela: enfoques didácticos sobre insectos en la educación básica

Thayline Rodrigues de Oliveira ORCID: https://orcid.org/0000-0002-4195-6995

Universidade Federal do Piauí, Brasil

E-mail: thaylinerodrigues@ufpi.edu.br

Luciana Barboza Silva

ORCID: https://orcid.org/0000-0002-7127-600X

Universidade Federal do Piauí, Brasil

E-mail: lubarbosabio@ufpi.edu.br

Ricardo de Jesus Fialho

ORCID: https://orcid.org/0000-0002-4593-569X

Universidade Federal do Piauí, Brasil

E-mail: fialhoricardo59@gmail.com

Acalene Gonçalveis de Oliveira ORCID: https://orcid.org/0000-0001-6720-3134

Universidade Federal de Pernambuco, Brasil

E-mail: acalene.ago@ufpe.br

Maria Luiza de Sousa Neta

ORCID: https://orcid.org/0000-0002-0321-1108

Universidade Estadual Santa Cruz, Brasil

E-mail: marialuizadesousa4@ gmail.com

Sandra Ribeiro da Silva

ORCID: https://orcid.org/0000-0002-9792-8803

Universidade Federal do Piauí, Brasil

E-mail: sandra21.rsilva@gmail.com

Vynicius Barbosa de Oliveira

ORCID: https://orcid.org/0000-0002-7544-4904

Universidade Federal do Pará, Brasil

E-mail:vynicius.oliveira@ig.ufpa.br

Camila Costa da Silva

ORCID: https://orcid.org/0000-0001-5100-6726

Universidade Federal do Piaú, Brasil

E-mail: camilacosta@ufpi.edu.br

Edineia da Silva Araújo

ORCID: https://orcid.org/0000-0002-5552-3410

Universidade Estadual Santa Cruz, Brasil

E-mail: araujo.es.bio@gmail.com

Josenir Teixeira Câmara

ORCID: https://orcid.org/0000-0001-7549-0575

Universidade Federal do Piauí, Brasil

E-mail: josenircamara@ufpi.edu.br

Bianca Fernandes dos Santos

ORCID: https://orcid.org/0000-0002-8856-5828

Universidade Federal do Piauí, Brasil

E-mail: biancafernandeshta@gmail.com

Michela Santos da Silva

ORCID: https://orcid.org/0000-0002-5781-9717

Universidade Federal do Piauí, Brasil

E-mail: michelasantos2013@gmail.com

Ana Clara Pereira da Silva

ORCID: https://orcid.org/0000-0002-5336-8568

Universidade Federal do Piauí, Brasil

E-mail: annaclara20012020@gmail.com

\section{Resumo}

A classe Insecta compreende o grupo de organismos com maior diversidade sobre a Terra, possuem ampla distribuição e riqueza de espécies sendo possível encontrá-los em todas as épocas do ano. Os insetos tornaram-se elementos de extrema importância, pois participam de todos os processos biológicos. Este trabalho objetivou promover conhecimentos a respeito dos insetos, por meio de realização de abordagens didáticas, a fim de despertar a 
participação e a curiosidades dos estudantes sobre os insetos. Nesse sentido, o projeto de extensão " Insetos na Escola", desenvolveu diversas atividades como modelos biológicos, caixas entomológicas, jogos, atividades de microscopia, com o intuito de informar sobre o papel e a relação dos insetos para meio ambiente e biodiversidade, os seres humanos, a agricultura e a economia. As atividades ocorreram semanalmente. Foi possível a interação com os alunos, eliminando dúvidas e curiosidades sobre os insetos e sua criação, permitindo a compreensão dos principais grupos de insetos encontrados em seu dia-a-dia. Os alunos puderam entender as características que cada grupo de inseto possui, relacionando-as com os diversos aspectos relativos ao modo de vida, comportamento e classificação dos mesmos, que facilitou associação entre teoria, despertando a curiosidade e o interesse dos alunos sobre o estudo dos insetos, além de ter se mostrado importante no processo de desenvolvimento da aprendizagem significativa dos educandos. Com as atividades desenvolvidas observou-se que o projeto serviu como uma ponte entre a academia e o meio social, constituindo-se como uma fundamental ferramenta no ensino de ciências e biologia.

Palavras-chave: Entomologia; Métodos alternativos; Material didático; Ensino remoto.

\begin{abstract}
The Insecta class comprises the group of organisms with the greatest diversity on Earth, they have a wide distribution and richness of species and it is possible to find them at all times of the year. Insects have become extremely important elements, as they participate in all biological processes. This work aimed to promote knowledge about insects, through the realization of didactic approaches, in order to awaken the participation and curiosity of students about insects. In this sense, the extension project "Insects at School", developed several activities such as biological models, entomological boxes, games, microscopy activities, in order to inform about the role and relationship of insects to the environment and biodiversity, beings human resources, agriculture and the economy. Activities took place weekly. Interaction with students was possible, eliminating doubts and curiosities about insects and their creation, allowing for an understanding of the main groups of insects found in their daily lives. Students were able to understand the characteristics that each group of insects has, relating them to the various aspects related to their way of life, behavior and classification, which facilitated the association between theory, arousing students' curiosity and interest in the study of insects, in addition to having shown to be important in the process of developing meaningful learning of students. With the activities developed, it was observed that the project served as a bridge between academia and the social environment, constituting a fundamental tool in the teaching of science and biology.
\end{abstract}

Keywords: Entomology; Alternative methods; Teaching material; Remote teaching.

\title{
Resumen
}

La clase Insecta comprende el grupo de organismos con mayor diversidad en la Tierra, tienen una amplia distribución y riqueza de especies y es posible encontrarlos en todas las épocas del año. Los insectos se han convertido en elementos extremadamente importantes, ya que participan en todos los procesos biológicos. Este trabajo tuvo como objetivo promover el conocimiento sobre los insectos, a través de la realización de enfoques didácticos, con el fin de despertar la participación y curiosidad de los estudiantes sobre los insectos. En este sentido, el proyecto de extensión "Insectos en la escuela", desarrolló diversas actividades como modelos biológicos, cajas entomológicas, juegos, actividades de microscopía, con el fin de informar sobre el papel y relación de los insectos con el medio ambiente y la biodiversidad, seres humanos, recursos humanos, agricultura y economía. Las actividades se llevaron a cabo semanalmente. La interacción con los estudiantes fue posible, eliminando dudas y curiosidades sobre los insectos y su creación, permitiendo conocer los principales grupos de insectos que se encuentran en su vida diaria. Los estudiantes pudieron comprender las características que tiene cada grupo de insectos, relacionándolas con los diversos aspectos relacionados con su forma de vida, comportamiento y clasificación, lo que facilitó la asociación entre teoría, despertando la curiosidad e interés de los estudiantes por el estudio de los insectos, además de haber demostrado ser importante en el proceso de desarrollo de un aprendizaje significativo para los estudiantes. Con las actividades desarrolladas, se observó que el proyecto sirvió de puente entre la academia y el entorno social, constituyendo una herramienta fundamental en la enseñanza de la ciencia y la biología.

Palabras clave: Entomología; Métodos alternativos; Material didáctico; Enseñanza a distancia.

\section{Introdução}

Os insetos são animais pertencentes ao filo Arthropoda e classe Insecta, constituem o táxon com maior diversidade sobre a Terra (Lopes \& Silva, 2019). Por apresentar abundância de espécies é possível encontrá-los em qualquer lugar e em quase todas as épocas do ano (Macêdo et al., 2016). Nesse sentido, os insetos é um grupo de extrema importância, pois participa de todos os processos ecológicos como ciclagem de nutrientes, polinização das plantas e dispersão de sementes dentre outros (Amaral et al., 2017).

No entanto, apesar de haver estudos pela comunidade científica sobre os insetos, observa-se que boa parte da população ainda os consideram como repulsivos ou nocivos à saúde, e pensamentos como estes promovem o declínio de várias 
espécies, juntamente com as ações de desmatamento e uso excessivo de agrotóxicos (Lopes \& Silva, 2019). Nesta perspectiva, ressalta-se a importância de trabalhos com os insetos, principalmente com o público da educação básica, pois são excelentes fermentas didáticas, demonstrativas e experimentais que ajudam a desmitificar informações erradas a respeito dos insetos, além de mostrar seus benefícios para nós seres humanos.

O ensino de Ciências envolve muito mais que memorização de conceitos e conteúdo, sendo necessário uma abordagem e práticas científicas que estabeleçam uma ponte, relacionando os conteúdos vistos em sala de aula com o cotidiano dos estudantes, tornando a aprendizagem significativa (Leal, 2020). O projeto de extensão visa impulsionar uma maior aproximação entre a Universidade e as escolas da rede básica de ensino e diferentes comunidades, e com isso, proporcionar conhecimentos a respeito dos insetos e sua relação com o homem, animais e o meio ambiente, incentivar a participação e a curiosidades dos estudantes sobre principais características e funções dos representantes desse grupo. O projeto utilizou modelos didáticos e caixas entomológicas, que são considerados facilitadores no processo de aprendizagem, pois aproxima da realidade aquilo que é visto em teoria. Modelos didáticos são aliados dos profissionais educação, uma vez que instiga os alunos a produzirem novos conhecimentos e fazerem assimilações. Vale ressaltar que o rendimento dos alunos aumenta de forma significativa quando se trabalha de forma interativa e participativa (Neckel, 2018; Santos; Silva; Lima, 2018).

Além disso, o projeto "Insetos na Escola" procura promover conhecimentos a respeito da classe Insecta, despertando interesses em crianças e adolescentes, e são essas novas informações que contribuirão para sua formação básica. Com a pandemia causada pelo SARS-CoV-2, o ambiente virtual foi de suma importância para o processo de aprendizagem e os educadores tiveram que se reinventar, utilizando principalmente ferramentas digitais, para promoção e continuidade do processo de ensino e aprendizagem. Nesse novo cenário há uma maior aproximação entre educadores e educandos (Soares et al., 2021). E diante da necessidade do distanciamento social, o projeto sofreu algumas alterações, continuando ao cronograma do projeto de forma remota, porém mantendo o vínculo com os alunos para o desenvolvimento das atividades. Diante do exposto, o presente estudo teve como objetivo construir e compartilhar conhecimentos sobre os insetos, por meio da realização de abordagens didáticas utilizando os recursos de multimidia, além de possibilitar a ampliação do conhecimento sobre a diversidade e distribuição do grupo, e assim, gradativamente alterar o padrão de conceituação acerca da Classe Insecta, dialogando com o público envolvido.

\section{Metodologia}

O projeto "Inseto na Escola" foi desenvolvido entre os anos de 2019 e 2020, na Unidade Escolar José Lustosa Elvas Filho, situada no município de Bom Jesus-PI, o projeto foi realizado por alunos do Curso de Ciências Biológicas do Campus Professora Cinobelina Elvas (CPCE), Universidade Federal do Piauí (UFPI) com a colaboração do grupo de Pesquisa Experimentação e Pesquisa em Manejo Integrado de Pragas (EPMIP) (Figura 1AB). 
Figura 1. Grupo de pesquisa EPMIP (A), Extensionista do projeto insetos na escola, alunos do curso de Ciências Biológicas (B).
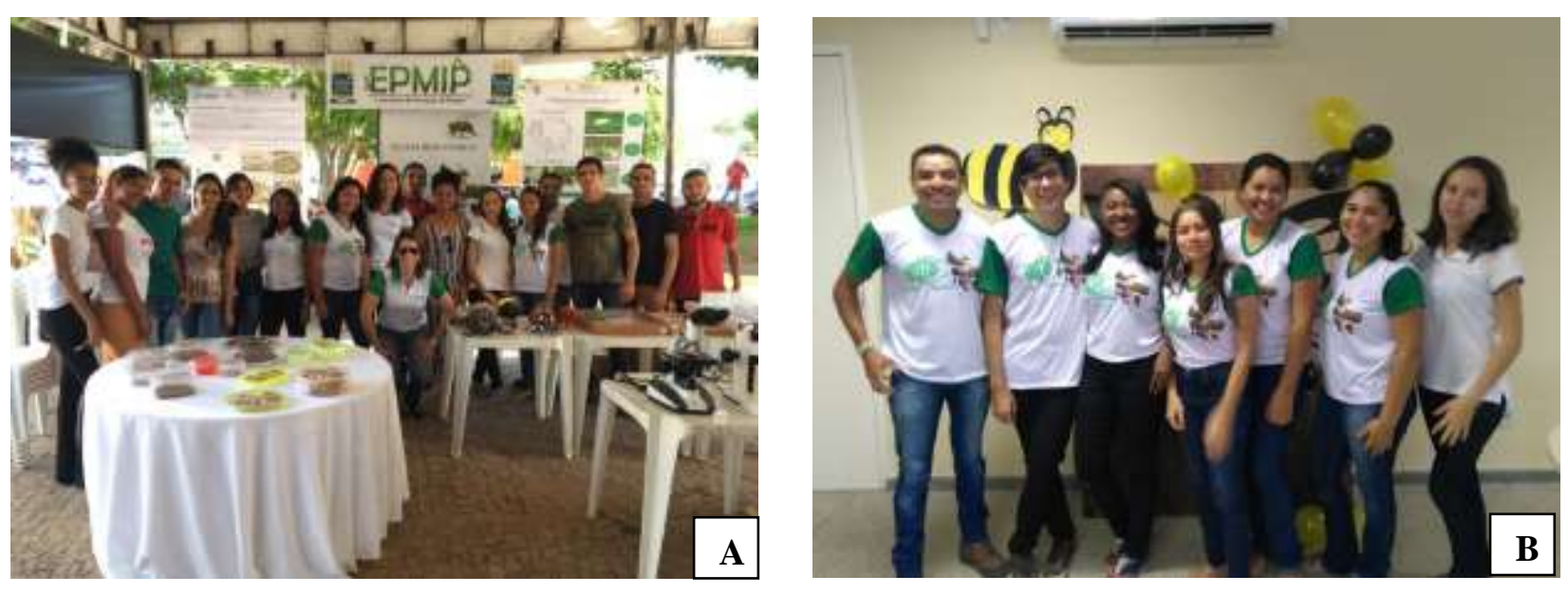

Fonte: Autores.

No ano de 2019 as atividades foram realizadas presencialmente. Os encontros ocorreram semanalmente através de agendamentos prévios com os professores e diretor da escola. As exposições ocorreram no horário de intervalo, em turno único (vespertino), de maneira a permitir a participação de todos os discentes. Os bolsistas responsáveis pela aplicação do projeto abordavam assuntos como: biodiversidade, importância dos insetos, características gerais das ordens e famílias, nomes populares, insetos polinizadores, insetos urbanos e sociais.

O material exposto na escola consistiu de caixas entomológicas compostas por exemplares de diferentes ordens de insetos, lâminas com estruturas corporais dos insetos, microscópio, lupa microscópica, banners, modelos didáticos, bandejas de criação das larvas, insetódromo. As amostras que compõem a coleção didática foram coletadas em campo pelos bolsistas do projeto. Os materiais coletados foram levados para o laboratório de zoologia localizado no CPCE/UFPI, onde foram triados, etiquetados e identificados em nível de ordem e famílias taxonômicas para posterior preparação das caixas entomológicas.

Em 2020, a pandemia desencadeada pelo novo coronavírus SARS-CoV-2, declarada pela Organização Mundial da Saúde (OMS), marcou o ano com grandes mudanças e adaptações em praticamente todas as áreas de atuação dos brasileiros.

Diante o momento pandêmico, às atividades do projeto deveriam continuar, mesmo que de forma não presencial. Sendo assim, muitas ações foram desenvolvidas para tentar reestabelecer o projeto em meio a pandemia. Inicialmente foi elaborado um questionário diagnóstico online, com perguntas objetivas e subjetivas afim verificar as dificuldades que os educandos estavam enfrentando com as atividades remotas. O questionário foi elaborado pelos extensionistas e posteriormente enviado aos discentes da escola onde o projeto foi realizado.

As atividades passaram a ser executadas de forma remota, por meio de plataformas de multimídia a saber: Google meet ${ }^{\circledR}$, instagram ${ }^{\circledR}$, homepage do Grupo de Pesquisa em Experimentação e Pesquisa em Manejo Integrado de Pragas EPMIP (www.epmip.ufpi.edu.br) e Whatsapp ${ }^{\circledR}$. As atividades foram divididas e realizadas da seguinte forma: apresentações em powerpoint sobre características gerais e importância dos insetos, insetos polinizadores, insetos urbanos, insetos alimentícios entre outros temas (Google meet $\left.{ }^{\circledR}\right)$; coleta de diferentes insetos, identificação de imagens com insetos, amostras de criações de insetos e debate e dúvidas de notícias relacionadas aos insetos (Grupo de Whatsapp®); postagens sobre curiosidades sobre os insetos (Instagram: @insetosnaescola, @epmip) e postagens diversas relacionadas aos insetos (homepage do grupo EPMIP). 


\section{Resultados e Discussão}

\subsection{Primeiro ano de realização do projeto}

Durante o desenvolvimento do projeto "Insetos na Escola" de forma totalmente presencial foram realizadas exposições (Figura 2) na Unidade Escolar José Lustosa Elvas Filho. As aplicações práticas ocorrem por meio de exposição dos materiais didáticos com utilização de caixas entomológicas, lâminas com estruturas corporais dos insetos, microscópio, lupa, modelos didáticos, banners dentre outros. Todas as atividades propostas despertaram a curiosidade dos estudantes em relação a temática envolvida, os insetos.

Das aplicações práticas que chamaram mais atenção dos estudantes, foram as caixas entomológicas compostas por exemplares de diferentes ordens de insetos, pois aguçou a curiosidade dos alunos pela diversidade de formas, tamanho e cores dos insetos encontradas em cada uma das ordens, além de reconhecimento de vários insetos que eles costumam visualizar no seu dia a dia ( Figura 2A). Com base no exposto, as atividades envolvendo os insetos são fundamentais para o processo de ensino e aprendizagem dos estudantes, pois proporcionam experiências a eles que podem ser muito diferentes da metodologia abordada em sala de aula, uma vez que, existe um contato mais próximo com os insetos, seja por meio de coleções entomológicas ou de exemplares didáticos, tais materiais promovem maior interatividade e estimulam os estudantes a aprender mais sobre estes animais (Baccin et al., 2020).

Outra atividade que despertou bastante interesse nos estudantes foi a visualização dos insetos em lupas microscópicas e visualização de estruturas corporais no microscópio (Figura 2B), onde foi possível que cada estudante visualizasse e entendesse as estruturas corporais expostas de cada inseto. Nesse contexto, o uso de metodologias alternativas para o ensino de ciências possibilita e incentiva uma participação efetiva dos estudantes, o que contribui não apenas para o processo de ensino e aprendizagem, mas também propicia a troca e produção de conhecimento, a criatividade, a curiosidade, além de despertar o caráter crítico e investigativo nos discentes (Guimaraes-Brasil et al., 2017).

Figura 2. Observação de caixas entomológicas (A) e visualização microscópica de estrutura corporais dos insetos (B).
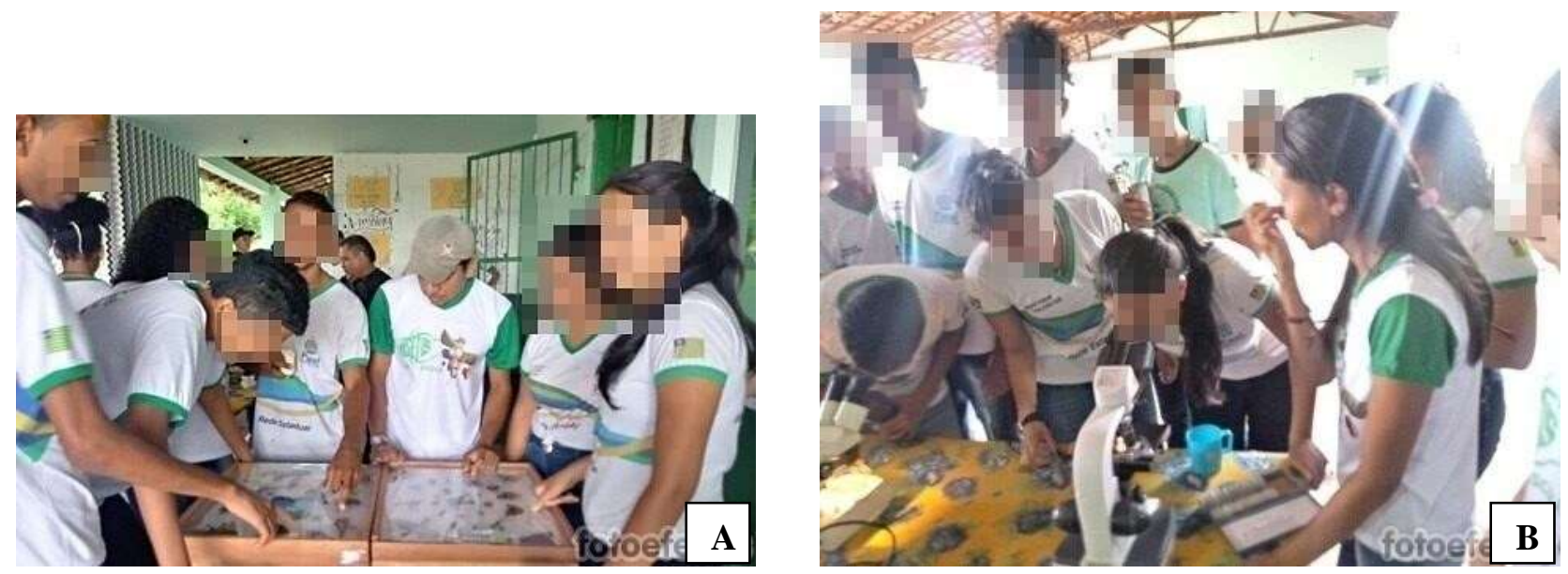

Fonte: Autores.

Durante realização do projeto na escola, foi possível perceber que, alguns estudantes não conseguiam distinguir os insetos de outros artrópodes, por exemplo, incluíam carrapatos e escorpiões na classe Insecta, e estes sistematicamente pertencem a classe Chelicerata (Figura 3A). Entretanto, com a realização de atividades práticas sobre os insetos, os alunos entenderam e conseguiram distinguir cada grupo de animal apresentado, além de relacioná-los com os diversos aspectos 
relativos ao seu modo de vida, comportamento e classificação, o que facilitou a compressão e identificação dos insetos estudados, proporcionando uma aprendizagem significativa dos alunos (Figura 3B).

Nesse sentido, Wardenski e Giannella (2017) destacam que um dos objetivos da educação é estimular o conhecimento sobre o ensino de ciências incluindo os insetos, com a finalidade de contribuir para a compreensão de seu papel nos diferentes ambientes onde são encontrados, além disso, o desenvolvimentos de projetos com a utilização dos insetos contribuem para diminuir a ideia negativa que muitos estudantes tem sobre eles, devido a associações com doenças e danos provocados a agricultura, para isso, a temática pode ser abordada desde as características que identificam cada insetos (anatomia, fisiologia e comportamento) até suas relações com os seres humanos, discutindo seus benefícios e esclarecendo os malefícios.

Figura 3. Explicação da diferença dos artrópodes (A), Observação das estruturas morfológicas dos insetos (B).
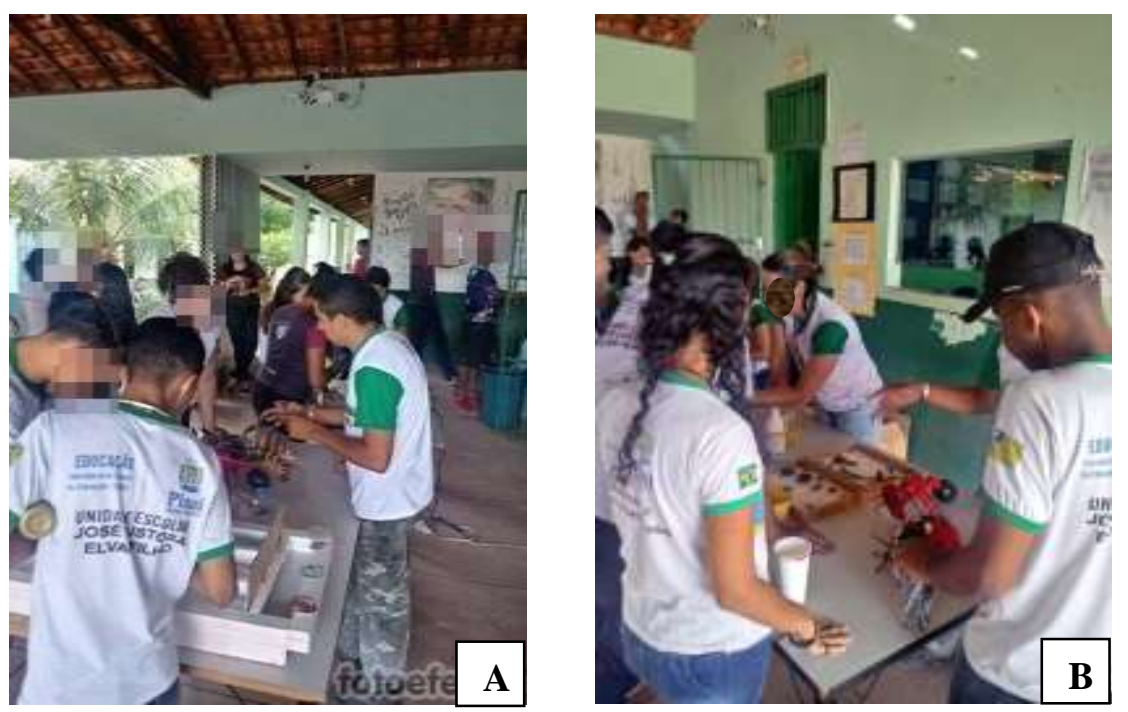

Fonte: Autores.

Além das atividades que foram desenvolvidas na escola, também foi realizada em Bom Jesus - PI no evento Dia C da Ciência na $17^{\mathrm{a}}$ Semana Nacional de Ciência e Tecnologia do Piauí (Figura 4) o evento foi aberto à comunidade interna (Figura 4A) e externa à UFPI (Figura 4B), que participou ativamente das atividades e na $4^{\mathrm{a}}$ edição do SaLiBom (Salão do Livro de Bom Jesus) evento realizado no Campus Professora Cinobelina Elvas (CPCE/UFPI) (Figura 5), o evento contou com a participação da comunidade, escolas de ensino fundamental e médio do município de Bom Jesus - PI, onde tivemos diversas atividades, dentre elas a observação das estruturas do insetos e visualização em caixa entomológicas (Figura 5A-B), pinturas no corpo com desenhos de insetos (Figura 5 C-D). 
Figura 4. Exposição do projeto insetos na escola no dia $\mathrm{C}$ da Ciência.
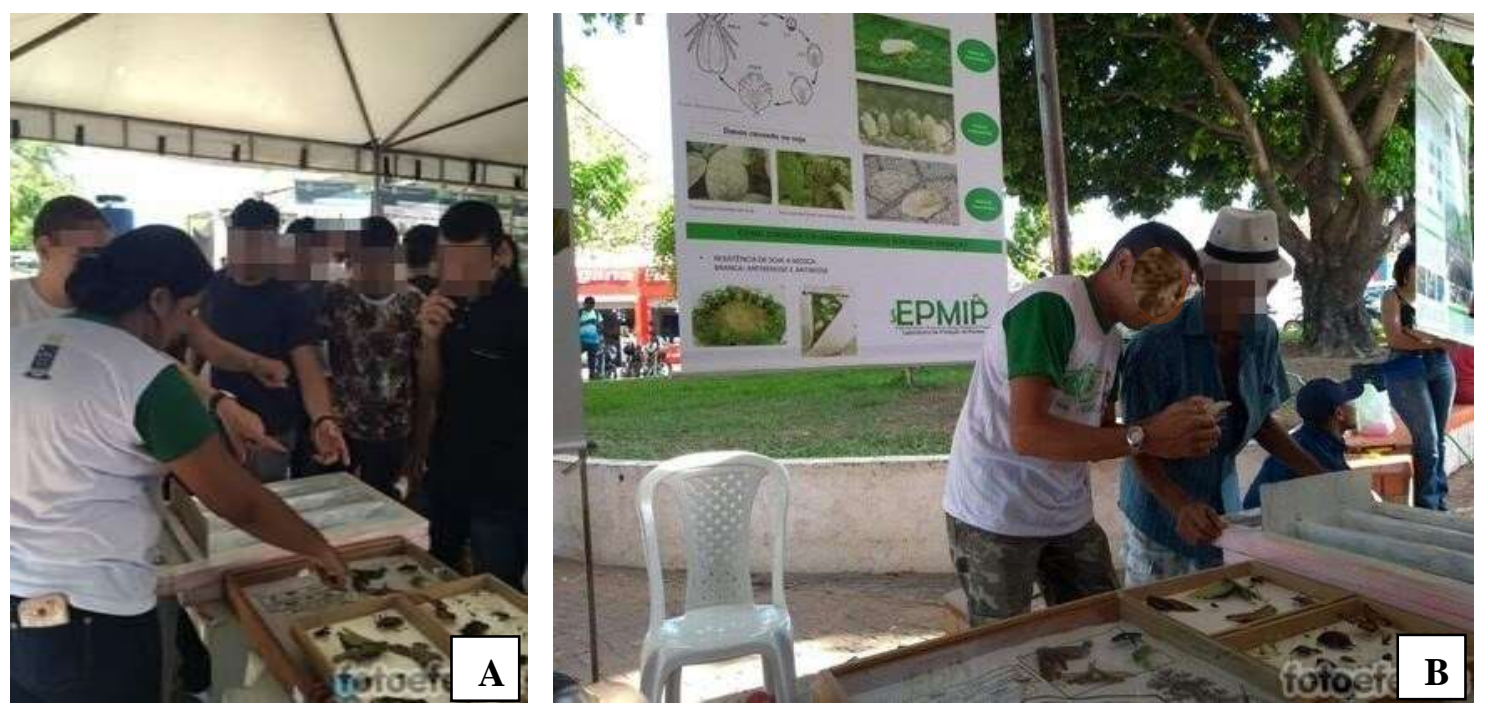

Fonte: Autores.

$\mathrm{Na}$ mostra, os integrantes do projeto apresentaram os trabalhos realizados durante todo ano na escola, proporcionando aos estudantes de diferentes escolas conhecer e entender um pouco mais sobre a fascinante classe Insecta. De acordo com Matos et al., (2009) a realização de trabalhos que trazem abordagens práticas para ensino, tem como objetivo consolidar o conhecimento teórico trabalhado por meio dos livros didáticos em sala de aula, além de quebrar o modelo "tradicionalista" e tornar o aluno um ser ativo no processo de ensino e aprendizagem. 
Figura 5. Exposição do projeto durante a $4^{\mathrm{a}}$ edição do Salão do Livro de Bom Jesus, o SaLiBom do Campus Professora Cinobelina Elvas (CPCE/UFPI).
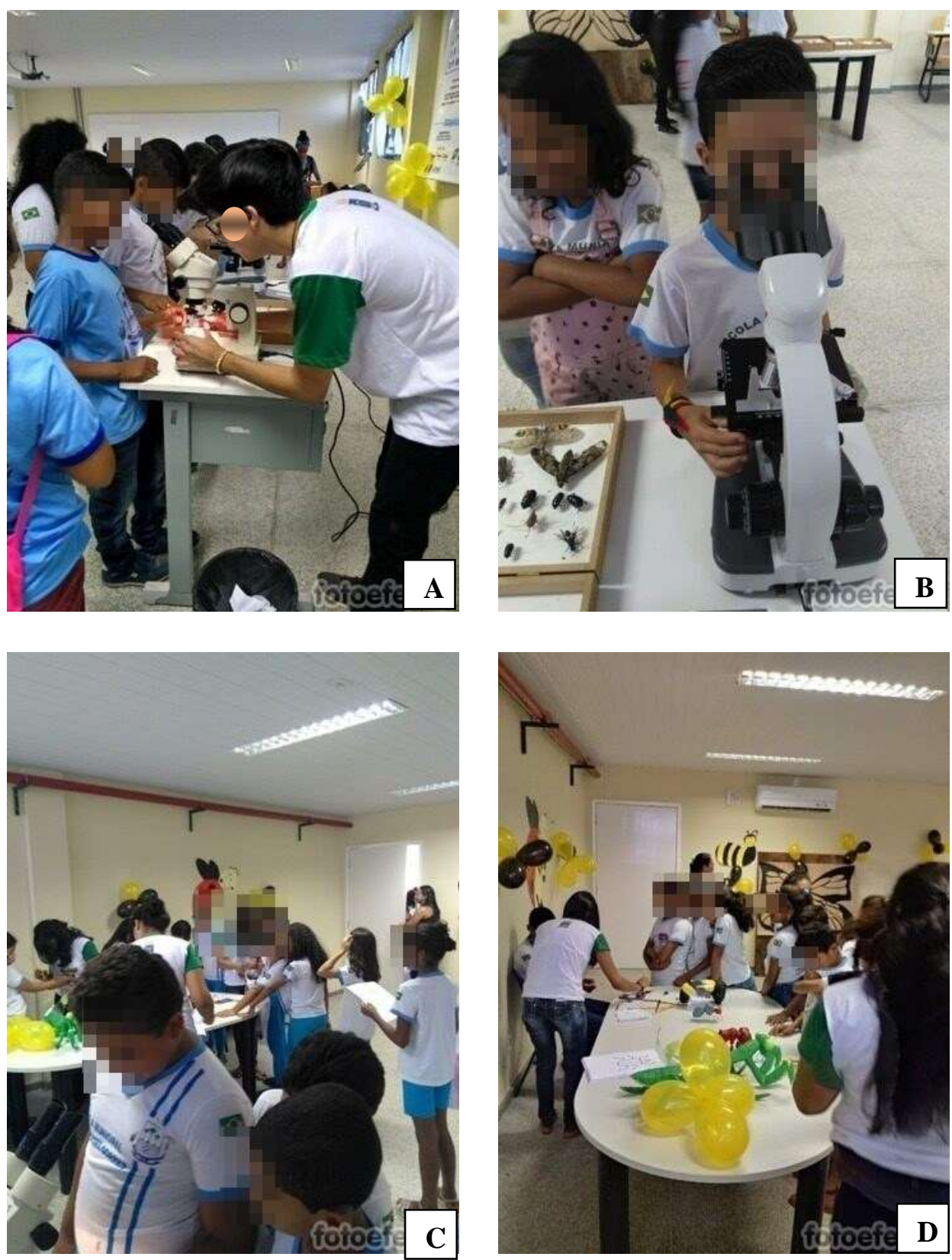

Fonte: Autores.

A partir deste contexto, os insetos são excelentes recursos didáticos, não apenas para a área de estudo da zoologia, mas em todos os campos das Ciências Biológicas, pois é notável a sua função interdisciplinar em diversos conteúdos, assim como sua importância ecológica e a relação com os seres humanos (Ferreira et al., 2020).

\subsection{Segundo ano de realização do projeto}

Diante a pandemia desencadeada pelo novo coronavírus SARS-CoV-2, o projeto de extensão "Insetos na Escola" precisou se reinventar. 
Todavia, a referida pandemia trouxe uma "ressignificação" para a educação, nos levando a repensar a educação e todos os seus processos. Com isso, os docentes e discentes tiveram que se adaptarem aos diversos aplicativos, programas e ferramentas utilizadas para socializar o conhecimento sobre diversos conteúdos (Pasini et al., 2020).

A continuação do projeto aconteceu por plataformas multimídias, onde os encontros foram realizados pelo Google meet (Figura B), onde explicou-se sobre quem são os insetos, onde podem ser encontrados, suas características, classificação e sobre sua importância para a sociedade, além da exposição da coleção entomológicas.

Figura 6. Atividades realizadas de forma remota: página do instagram ${ }^{\circledR}$ (Figura A) e palestra no google meet ${ }^{\circledR}$ (Figura 6B).

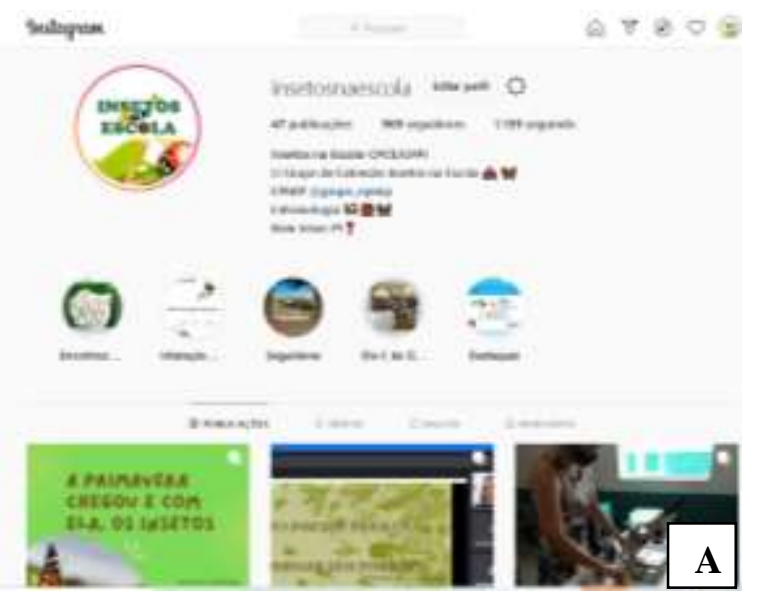

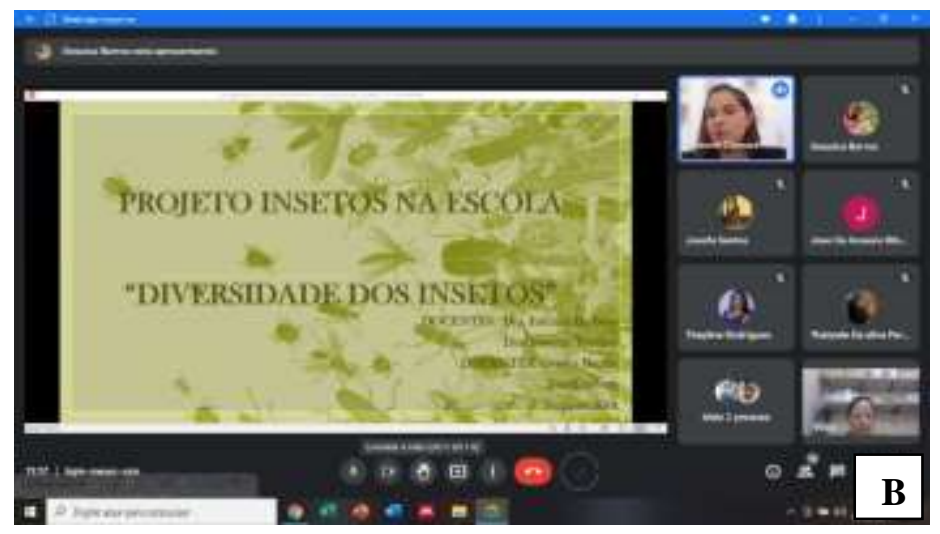

Fonte: Autores.

Houveram, também, as realizações de várias dinâmicas utilizando o grupo criado no aplicativo WhatsApp, onde todos os alunos puderam participar, seja na identificação das ordens dos insetos que apareciam nas imagens disponibilizadas, seja nas discussões e dúvidas sobre notícias visualizadas na internet. Durante a abordagem dos temas e apresentação do projeto, foi possível perceber a interação dos alunos, onde estes frequentemente fizeram questionamentos a respeito dos insetos

Ainda utilizando o aplicativo WhatsApp ${ }^{\circledR}$, textos informativos e postagens da página do instagram ${ }^{\circledR}$ (Figura 6A) e da Homepage do grupo EPMIP eram divulgados e discutidos, além dos alunos erem estimulados a postarem diariamente fotos de insetos que encontravam em seu dia-a-dia, onde os mesmos questionavam sobre curiosidades sobre os insetos encontrados. Nesse sentido, percebe-se a importância de se trabalhar a temática dos insetos para o público escolar associando-a com o cotidiano do discente, visto que este tema faz parte do conteúdo abordado em disciplinas presentes na matriz curricular, o que contribui para melhor entendimento dos assuntos abordados pelos professores (Araújo et al., 2019).

A partir desses pressupostos, cabe aos educadores a missão de transformar o ensino "tradicionalista", levando metodologias diferentes e ativas para a escola como atividades lúdicas e visuais para os alunos, de maneira que os estudantes tenham contato com diferentes atividades e no caso dos insetos diversos grupos existentes (Amaral et al., 2017).

Também foram produzidos vídeos explicando os diversos aspectos relacionados aos insetos, abordando as características do grupo, o processo de conservação dos insetos, criação do inseto Tenebrio molitor e sua importância para a alimentação. Estes vídeos foram disponibilizados para os estudantes participantes do projeto.

Essa atividade despertou bastante interesse nos estudantes, onde os mesmos participaram ativamente por meio de questionamentos sobre a criação do T. molitor, como se alimentam e para que são usados. No contexto educacional, abordar os insetos no ensino de ciências e biologia possibilita aos estudantes a construção de conhecimentos, argumentação, o criticismo bem como o habito investigativo, o que na maioria das vezes podem ser ausentes no processo de ensino-aprendizagem. Além 
disso, oferecem às docentes oportunidades de conhecer novos métodos ativos e incorporá-los em suas aulas diárias (Da Silva \& Lima, 2018).

\section{Considerações Finais}

Com as atividades presenciais, foi possível verificar que as exposições de diversos recursos para proporcionar o ensino de insetos trouxeram resultados de aprendizado positivo e de significância, além envolver os educandos na troca e produção do conhecimento acerca do grupo. E apesar das dificuldades enfrentadas com a pandemia causada pelo Covid-19 e do distanciamento social para evitar o contágio e a propagação do vírus, foi possível dar continuidade às atividades com os alunos da educação básica, utilizando recursos multimídias e apresentando-lhe diversos fatos e curiosidades sobre a classe Insecta, além da participação ativa dos alunos por meio de discussões e dúvidas, bem como pelo compartilhamento das suas vivências do dia-a-dia que envolviam os insetos.

Independentemente do modo (presencial ou remoto) que o projeto foi desenvolvido afim proporcionar o conhecimento acerca dos insetos aos alunos da educação básica, os insetos se mostraram excelentes como recurso didático, propiciando aos educandos a associação da teoria aprendida em sala de aula com seu cotidiano, melhorando significativamente o processo de ensino e aprendizagem.

Diante dos resultados obtidos com as atividades do projeto e da complexidade do momento em que passamos, devido à pandemia, seria de suma importância a continuação de trabalhos remotos e expansão do projeto a mais escolas da educação básica, proporcionando assim mais conhecimento e interação com os alunos.

\section{Agradecimentos}

Agradecemos a UFPI, PIBEX e a Secretaria Estadual de Educação do Piauí pelo apoio para a realização desse projeto.

\section{Referências}

Amaral, I. S. et al. (2017). A importância do resgate dos conhecimentos prévios e atividades práticas no ensino sobre insetos”. Revista Educar Mais, 1(1).

Araújo, R. C. S. et al. (2019). Entomologia na escola: recursos práticos para o ensino sobre insetos. Revistaea.org, (68), 1-8.

Baccin, K. M. S. et al. (2020). Os Insetos e a Ciência na Escola: Estratégias de Ensino. Scientia Cum Industria, 8(3), 13-16.

Ferreira, G. S. et al. (2020). Um Estudo sobre a Utilização da Vespa Parasitoide Melittobia (Hymenoptera: Eulophidae) como Recurso Didático para o Ensino de Insetos. Revista Insignare Scientia, 3(5). 1-19.

Guimaraes-Brasil, M. O. et al. (2017). Construção de caixas entomológicas como ferramenta ao ensino-aprendizagem em cursos técnicos de agrárias. Holos, $1(33)$.

Leal, S. C. (2020). Uso de metodologias ativas no ensino de entomologia no ensino médio. Tese (Ensino de Biologia em Rede Nacional) - Universidade Federal da Paraíba, João Pessoa.

Lopes, B. S., \& Silva, N.A. (2019). Entomologia na escola: o que os estudantes pensam sobre os insetos e como utilizá-los como recurso didático? Revista eletrônica de educação, 14, 1-20.

Macêdo, M. V. et al. (2016). Ensinar e aprender Ciências e Biologia com os insetos. In: Da-Silva, E. R., Passos, M. I. S., Aguiar, V. M., Lessa, C. S. S. \& Coelho, L. B. N. (eds.) - Anais do III Simpósio de Entomologia do Rio de Janeiro. Universidade Federal do Estado do Rio de Janeiro (UNIRIO), $12-23$.

Matos, C. H. C. et al. (2009). Utilização de Modelos Didáticos no Ensino de Entomologia. Revista de biologia e ciências da Terra, 9(1), 19-23.

Neckel, C. (2018). Modelos didáticos para ensino de Ciências e Biologia: cálculo renal. In: V Congresso Nacional de educação- V Conedu, 2018, Campina Grande. Anais, Realize Editora.

Da Silva, T. V., \& Lima, K. E. C. (2018). Etnoentomologia: percepção dos alunos do ensino fundamental sobre os insetos e suas importâncias. In: V Congresso Nacional de educação-VCONEDU, Recife. Anais.

Pasini, C. G. D, Carvalho, E, \& Almeida, L. H. C. (2020). A educação híbrida em tempos de pandemia: algumas considerações. Universidade Federal de Santa Maria. 
Research, Society and Development, v. 10, n. 16, e508101623081, 2021

(CC BY 4.0) | ISSN 2525-3409 | DOI: http://dx.doi.org/10.33448/rsd-v10i16.23081

Santos, R. O, Silva, P. S, \& Lima, J. L. S. (2018). Modelo didático como recurso para o ensino de ciências: sua influência como ferramenta facilitadora no processo de ensino aprendizagem. Revista Vivências em Ensino de Ciências. 2(3), 177-185.

Da Silva, T. V., \& Lima, K. E. C. (2018). Etnoentomologia: percepção dos alunos do ensino fundamental sobre os insetos e suas importâncias. In: V Congresso Nacional de educação-VCONEDU, Anais. Recife.

Soares, M. D. et al. (2021). Ensino de Biologia em tempos de pandemia: criatividade, eficiência, aspectos emocionais e significados. Revista Ibero-Americana de Humanidades, Ciências e Educação. 7(2).

Wardenski, R, De F., \& Giannella, T. R. (2017). Insetos no ensino de ciências: objetivos, abordagens e estratégias pedagógicas. In: XI Encontro Nacional de Pesquisa em Educação em Ciências XI ENPEC,2017, Florianópolis. Anais. 